\title{
Oral Health in Parkinson's Disease: How Long are we Going to Neglect it?
}

\author{
Jacqueline Fernandes do Nascimento ${ }^{1}$, Marco Orsini ${ }^{2 *}$, Antônio Marcos da Silva Catharino ${ }^{1}$, Valéria \\ Camargo Silveira ${ }^{1}$, Angélica Sabino Rodrigues ${ }^{1}$, Alair Pedro Ribeiro ${ }^{3}$, Bruna Velasques ${ }^{3}$, Mariana \\ Gangora $^{3}$, Adalgiza Mafra Moreno ${ }^{1}$, Carlos Eduardo Cardoso ${ }^{4}$, Marcos RG de Freitas ${ }^{3}$, Acary Souza Bulle \\ Oliveira ${ }^{5}$ and Marco Antonio Araujo Leite ${ }^{6}$
}

${ }^{1}$ Undergraduated in Pos-Graduation Program in Neurology and Neuroscience - Federal Fluminense University, Brazil

${ }^{2}$ Vassouras University - UV and Iguaçu University - UNIG, Brazil

${ }^{3}$ Rio de Janeiro Federal University - UFR, Brazil

${ }^{4}$ Vassouras University - UV - Rio de Janeiro Brazil

${ }^{5}$ São Paulo Federal University- Unifesp, SP, Brazil

${ }^{6}$ Pos-Graduation Program in Neurology and Neuroscience - Federal Fluminense University, Brazil

*Corresponding author: Marco Antônio Araujo Leite, Pos-Graduation Program in Neurology and Neuroscience - Federal Fluminense Univeristy - Hospital University Antônio Pedro - HUAP.
Received Date: July 06, 2021

Published Date: July 21, 2021

\section{Short Communication}

Parkinson's Disease is a common neurological disorder characterized by a depletion of dopaminergic transmission at the level of the basal ganglia, impairing automatic movements, in speed and amplitude [1]. However, non-motor symptoms such as cognitive, autonomic, sleep-related and sensory dysfunctions are often reported. A subgroup of non-motor symptoms, oropharyngeal problems, also affects these patients in ways that greatly deteriorate quality of life.Patients with PD present several changes regarding injuries to the oral cavity. A typical finding is the presence of sialorrhea and, undoubtedly, alterations in the symbiosis of the oral flora [2]. Current studies have assessed the excessive prevalence of sialorrhea in (PD), in addition to its changes between "ON" and OFF "conditions over time and impact on health-related quality of life [3]. This symptom was directly related to the duration and severity of PD, more frequent in males and associated with dysphagia, hypomimia and autonomic dysfunction. Sialorrhea is more frequent in PD patients than controls, worsened in the "OFF" condition and after $\sim 2$ years of follow-up. PD are at risk for developing oral health injuries that can exacerbate or be exacerbated by other non-motor symptoms, such as mental health and dysphagia. These changes can decline in quality of life and even increases the risk of death by aspiration pneumonia [4].

The oral microbiota of patients with PD is compared, through numerous studies, with a control group composed of subjects following specific inclusion and exclusion criteria. In these studies, differences in the diversity and abundance of bacterial rates were identified. A significant increase in the abundance of opportunistic oral pathogens was detected in patients with PD. Such microorganisms can cause specific diseases and, in some cases, decrease the survival. The oral microbiome deserves additional research regarding its connection to $\mathrm{PD}$ and its biomarker potential [5,].

A study conducted by Mihaila (2019) [6] and collaborators, aimed to identify changes in the function and microbiome of the upper and lower gastrointestinal tract that have been documented in Parkinson's disease (PD). The authors identify sensitive and specific biomarkers of changes in the oral microbiome of early stage PD through shotgun metatranscriptomic profiling. The authors recruited 48 PD subjects and 36 age- and gender-matched healthy controls. All individuals covered in the survey completed detailed assessments of motor, cognitive, balance, gait, autonomic, and chemosensory (smell and taste) functions to determine their disease stage. The researchers obtained a saliva sample for profiling of microbial RNA and host mRNA using next generation sequencing. As a result, no differences in overall alpha and beta diversity between subject groups. However, changes in specific microbial taxa were observed, including primarily bacteria, but also yeast and phage. Nearly half findings were consistent with prior studies 
in the field obtained through profiling of fecal samples, with others representing highly novel candidates for detection of early stage PD. These results suggest that the oral microbiome may represent a highly-accessible and informative microenvironment that offers new insights into the pathophysiology of the early stage PD.

Jeter (2018) [7] and his team defined a module created to update dental health professionals and educating dental students on the oral health needs of patients with PD, with an emphasis on interprofessional collaboration and evidence-based dentistry. This model may also benefit other health professionals such as, neurologists, who deal with PD. Guidance for patients and family members about the frequency of oral health reviews is needed, as well as early resolution of problems in this large cavity, which can improve or impair the quality of life of this patients. A deep knowledge of the microbiota of patients with PD can serve as a basis for the development of new researches that involve not only a targeted treatment, but also a diffusion in the pathophysiology of this complex disease.

\section{Acknowledgement}

None.

\section{Conflict of Interest}

No conflict of interest.

\section{References}

1. Umemoto G, Furuya H (2020) Management of Dysphagia in Patients with Parkinson's Disease and Related Disorders. Intern Med 59(1): 7-14.

2. Shen L (2020) Gut, oral and nasal microbiota and Parkinson's disease. Microb Cell Fact 19(1): 50.

3. Rascol O, Negre-Pages L, Damier P, Delval A, Derkinderen P, et al. (2020) Excessive buccal saliva in patients with Parkinson's disease of the French COPARK cohort. J Neural Transm (Vienna) 127(12): 1607-1617.

4. Rozas NS, Sadowsky JM, Jones DJ, Jeter CB (2017) Incorporating oral health into interprofessional care teams for patients with Parkinson's disease. Parkinsonism Relat Disord 43: 9-14.

5. Pereira PAB, Aho VTE, Paulin L, Pekkonen E, Auvinen P, et al. (2017) Oral and nasal microbiota in Parkinson's disease. Parkinsonism Relat Disord 38: 61-67.

6. Mihaila D, Donegan J, Barns S, LaRocca D, Du Q, et al. (2019) The oral microbiome of early stage Parkinson's disease and its relationship with functional measures of motor and non-motor function. PLoS One 14(6): e0218252.

7. Jeter CB, Rozas NS, Sadowsky JM, Jones DJ (2018) Parkinson's Disease Oral Health Module: Interprofessional Coordination of Care. MedEdPORTAL 14: 10699. 